\title{
Biological characteristics of intratumoral [F-18]-fluoromisonidazole distribution in a rodent model of glioma
}

\author{
TOSHIYUKI HATANO ${ }^{1,2}$, SONGJI ZHAO ${ }^{3,4}$, YAN ZHAO ${ }^{3,4}$, KEN-ICHI NISHIJIMA ${ }^{3,4}$, NORIHITO KUNO ${ }^{2}$, \\ HIROKO HANZAWA ${ }^{2}$, TAKESHI SAKAMOTO ${ }^{2}$, NAGARA TAMAKI ${ }^{4}$ and YUJI KUGE $^{1}$ \\ ${ }^{1}$ Central Institute of Isotope Science, Hokkaido University, Sapporo; ${ }^{2}$ Central Research Laboratory, \\ Hitachi Ltd., Kokubunji, Tokyo; Departments of ${ }^{3}$ Tracer Kinetics and Bioanalysis, and ${ }^{4}$ Nuclear Medicine, \\ Graduate School of Medicine, Hokkaido University, Sapporo, Japan
}

Received November 9, 2012; Accepted December 18, 2012

DOI: 10.3892/ijo.2013.1781

\begin{abstract}
Accurate imaging to identify hypoxic regions in tumors is key for radiotherapy planning. [F-18]-fluoromisonidazole ([F-18]-FMISO) is widely used for tumor hypoxia imaging and has the potential to optimize radiotherapy planning. However, the biological characteristics of intratumoral [F-18]-FMISO distribution have not yet been fully investigated. In hypoxic cells, the hypoxia-inducible factor-1 (HIF-1) target proteins that induce cellular proliferation and glucose metabolism, glucose transporter-1 (Glut-1) and hexokinase-II (HK-II), are upregulated. In this study, we determined the intratumoral distribution of [F-18]-FMISO by autoradiography (ARG) and compared it with pimonidazole uptake, expression of Glut-1, tumor proliferative activity (Ki-67 index) and glucose metabolism ([C-14]2-fluoro-2-deoxy-Dglucose uptake; [C-14]-FDG) in a glioma rat model. Five C6 glioma-bearing rats were injected with [F-18]-FMISO and [C-14]-FDG. After $90 \mathrm{~min}$, the rats were injected with pimonidazole and $60 \mathrm{~min}$ later, the rats were sacrificed and tumor tissues were sectioned into slices. The adjacent slices were used for ARG and immunohistochemical (IHC) analyses of pimonidazole, Glut-1 and Ki-67. [F-18]-FMISO ARG images were divided into regions of high [F-18]-FMISO uptake (FMISO+) and low [F-18]-FMISO uptake (FMISO-). Pimonidazole and Glut-1 expression levels, Ki-67 index and [C-14]-FDG distribution were evaluated in the regions of interest (ROIs) placed on FMISO+ and FMISO-. [F-18]-FMISO distribution was generally consistent with pimonidazole distribution. The percentage of positively stained areas (\% positive) of Glut-1 in FMISO+ was significantly higher compared to FMISO-
\end{abstract}

Correspondence to: Professor Songji Zhao, Department of Tracer Kinetics and Bioanalysis, Graduate School of Medicine, Hokkaido University, Kita 15 Nishi 7, Kita-ku, Sapporo 060-8638, Japan E-mail: zsi@med.hokudai.ac.jp

Key words: [F-18]-fluoromisonidazole, tumor hypoxia, cellular proliferation, glucose metabolism
$(24 \pm 8 \%$ in FMISO+ and $9 \pm 4 \%$ in FMISO-; $\mathrm{P}<0.05)$. There were no significant differences in Ki-67 index and [C-14]-FDG uptake between FMISO+ and FMISO- (for Ki-67, 10 $\pm 5 \%$ in FMISO+ and $12 \pm 5 \%$ in FMISO-, $\mathrm{P}=\mathrm{ns}$; for [C-14]-FDG, $1.4 \pm 0.3 \% \mathrm{ID} / \mathrm{g} / \mathrm{kg}$ in $\mathrm{FMISO}+$ and $1.3 \pm 0.3 \% \mathrm{ID} / \mathrm{g} / \mathrm{kg}$ in FMISO-, P = ns). Intratumoral [F-18]-FMISO distribution reflected tumor hypoxia and expression of the hypoxia-related gene product Glut-1; it did not, however, reflect tumor proliferation or glucose metabolism. Our findings help elucidate the biological characteristics of intratumoral [F-18]-FMISO distribution that are relevant to radiotherapy planning.

\section{Introduction}

Hypoxia develops in solid tumors due to the insufficiency of oxygen diffusion (chronic hypoxia) and blood perfusion (acute hypoxia) (1). Chronic hypoxia is the result of the long distance of tumor cells from the nearest blood vessel. Acute hypoxia is the result of fluctuating flow in blood vessels. Tumor cells in a hypoxic environment are more resistant to radiation damage than those in a normal oxygen environment, since radiation damage sensitivity depends on the oxygen concentration at the time of irradiation (2). Hypoxic regions are also related to tumor malignancy and proliferation (3-5). Therefore, hypoxia imaging can provide useful information for radiotherapy planning, including intensity-modulated radiation therapy (IMRT), and may also be a useful prognostic tool. [F-18]-Fluoromisonidazole ([F-18]-FMISO) is the most widely used positron emission tomography (PET) tracer for the imaging of tumor hypoxia. [F-18]-FMISO is a 2-nitroimidazole compound, which is reduced by nitroreductase enzymes in a hypoxic environment and trapped in hypoxic tumor cells. The hypoxia probe [F-18]-FMISO and the hypoxia marker pimonidazole are imidazole derivatives and they accumulate in similar regions in tumors (6,7). [F-18]-FMISO imaging of hypoxia may enable the optimization of radiotherapy planning and the prediction of radiotherapy outcome (8-10). Furthermore, hypoxia imaging using nitroimidazole has been used for patient selection for hypoxia-modifying treatments, including high-oxygen-content gas breathing and nitrometric radiation sensitizers $(11,12)$. 
Aside from oxygen concentration, biological characteristics related to hypoxia have also been reported to affect radiosensitivity and radiotherapy outcome $(4,13,14)$. Thus, it is important to elucidate the relationship between [F-18]-FMISO distribution and biological characteristics. However, the biological characteristics of intratumoral [F-18]-FMISO distribution have not yet been fully investigated. Extensive studies have been carried out on gene/protein expressions in hypoxic regions. Genes inducing glucose metabolism are upregulated in a hypoxic environment by hypoxia-inducible factor-1 (HIF-1) transcription factor $(15,16)$. HIF-1 target proteins include the glucose transporter-1 (Glut-1) and hexokinase-II (HK-II). HIF-1 and Glut-1 have been used as endogenous hypoxia markers to predict the response to radiotherapy (17). The expression of Glut- 1 and the functional activity of HK-II correlate with glucose metabolism in malignant tumors $(18,19)$. HIF-1 also upregulates genes inducing cellular proliferation (20) and knockdown of HIF-1 $\alpha$ results in a decrease in cellular proliferation rate in vivo (16). We therefore compared the intratumoral [F-18]-FMISO distribution with Glut-1 and $\mathrm{Ki}-67$ expression and with [C-14]2-fluoro-2-deoxy-D-glucose ([C-14]-FDG) distribution in a rat glioma model, in order to gain insight into the biological characteristics of intratumoral [F-18]-FMISO distribution that is relevant to radiotherapy planning.

\section{Materials and methods}

Animal studies. The experimental protocol was approved by the Laboratory Animal Care and Use Committee of Hokkaido University. Eight-week-old male Wistar King Aptekman/Hok (WKAH) rats (supplied by Japan SLC, Inc., Hamamatsu, Japan) were inoculated with a suspension of allogenic C6 rat glioma cells $\left(2 \times 10^{6}\right.$ cells $\left./ 0.2 \mathrm{ml}\right)$ into the left calf muscle to generate a rat glioma model (21). The rats were allowed free access to water and laboratory chow until the day before the experiment. Nine days after the tumor inoculation, when the tumors had reached $1-2 \mathrm{~cm}$ in diameter, the rats were fasted overnight $(n=5)$. Under diethyl ether anesthesia, the tail vein was injected with a mixture of 29-37 MBq of [F-18]-FMISO and $370-493 \mathrm{kBq}$ of [C-14]-FDG. [F-18]-FMISO (specific activity, $45-70 \mathrm{MBq} / \mathrm{nmol}$ ) was synthesized as previously described (22,23). Universally labeled [C-14]-FDG (specific activity, $11.1 \mathrm{GBq} / \mathrm{mmol}$ ) in sterile saline was purchased from American Radiolabeled Chemicals, Inc. Eighty minutes after the injection of [F-18]-FMISO and [C-14]-FDG, the rats were anesthetized with pentobarbital $(50 \mathrm{mg} / \mathrm{kg}$ body weight, intraperitoneally). Ten minutes after the injection, the rats were injected into the tail vein with pimonidazole (Hypoxyprobe-1; HPI Inc., Burlington, MA, USA) at a dose of $60 \mathrm{mg} / \mathrm{kg}$ body weight. Sixty minutes after the pimonidazole injection the animals were sacrificed and the tumors were quickly excised. The calf muscles were excised with the tumors. Each specimen was then sectioned to obtain two adjacent 3-5-mm slices. One of the two slices was embedded in Tissue-Tek medium (Sakura Finetechnical Co., Ltd.) and frozen in isopentane/dry ice for autoradiography (ARG) and immunohistochemical (IHC) analyses. The remaining slice was prepared as the formalinfixed, paraffin-embedded specimens for IHC analyses of pimonidazole.
Dual-tracer ARG. The frozen samples were cut into $20-\mu \mathrm{m}$ and 5- $\mu \mathrm{m}$ adjacent sections with a CM3050-Cryostat (Leica Microsystems) at $-20^{\circ} \mathrm{C}$. Tumor sections $(20 \mu \mathrm{m}$ and $5 \mu \mathrm{m})$ were prepared for ARG. The radioactivity of [F-18]-FMISO was measured in $20-\mu \mathrm{m}$ sections. The radioactivity of [C-14]-FDG was measured in 5- $\mu \mathrm{m}$ sections. [C-14]-FDG ARG images of $20-\mu \mathrm{m}$ sections were used for superimposing. Four 5- $\mu \mathrm{m}$ tumor sections were prepared for IHC and hematoxylin-eosin (HE) staining. The $20-\mu \mathrm{m}$ and $5-\mu \mathrm{m}$ tumor sections were placed in a phosphor image plate cassette with a set of calibrated standards (24), and the specimens were exposed to phosphor imaging plates (Fuji Imaging Plate, Fuji Photo Film Co., Ltd.) overnight to detect the distribution of [F-18]-FMISO. During ARG exposure, a polypropylene film was set between the phosphor imaging plate and a set of tumor sections and calibrated standards to block the $\beta$-rays from [C-14]-FDG. Two days later, following the decay of [F-18]-FMISO, the same tumor sections and calibrated standards were exposed to a phosphor imaging plate for 14 days to detect the distribution of [C-14]-FDG. The HE staining of the sections was carried out to exclude necrotic/apoptotic regions in the regions of interest (ROIs) on the autoradiograms. The ARG images obtained were analyzed using a computerized imaging analysis system (BAS 5000 Bio-Imaging Analyzer; Fuji Photo Film Co., Ltd.). The ARG resolution of BAS 5000 was $25 \mu \mathrm{m}$. The radioactivity in each ROI was expressed as the percentage activity of injected dose (ID) per gram of tissue following normalization to the animal's weight $(\% \mathrm{ID} / \mathrm{g} / \mathrm{kg})$, with the hypothesis that the tissue density is $1 \mathrm{~g} / \mathrm{cm}^{3}(24,25)$.

Immunohistochemistry. The experimental conditions of IHC were set to be identical using an automated staining system (Autostainer Plus, Dako) (26). The uptake of pimonidazole and the expression of Glut-1 and Ki-67 were examined in the frozen sections. Four adjacent tumor sections were used for pimonidazole, Glut-1, Ki-67, or HE staining. For pimonidazole staining, following rehydration, the slides were immersed in a citrate buffer solution (pH 6.0) and heated for $15 \mathrm{~min}$ at $121^{\circ} \mathrm{C}$ to retrieve the antigen. Subsequently, endogenous peroxidase activity was blocked for $10 \mathrm{~min}$ in $0.3 \%$ hydrogen peroxide. Thereafter, the slides were incubated with Hyproxyprobe-1 MAb1 (HPI Inc.) for $30 \mathrm{~min}$ at $37^{\circ} \mathrm{C}$, and then with biotinconjugated $\mathrm{F}\left(\mathrm{ab}^{\prime}\right) 2$ for $15 \mathrm{~min}$ at $37^{\circ} \mathrm{C}$. Following incubation with the antibodies, the bound antibody complex was visualized by incubation with streptavidin and 3,3-diaminobenzidine tetrahydrochloride. For Glut-1 and Ki-67 staining, the slides were immersed in a target retrieval solution ( $\mathrm{pH} 9.0$; Nichirei) and heated for $10 \mathrm{~min}$ at $95^{\circ} \mathrm{C}$ following rehydration. Endogenous peroxidase activity was then blocked for $10 \mathrm{~min}$ in $0.3 \%$ hydrogen peroxide. Thereafter, the slides for Glut-1 staining were incubated with anti-Glut-1 (Abcam) for $30 \mathrm{~min}$ at $37^{\circ} \mathrm{C}$, and then with anti-rabbit immunoglobulins/biotinylated (Dako) for $30 \mathrm{~min}$ at $37^{\circ} \mathrm{C}$. The slides for Ki-67 staining were incubated with an anti-Ki-67 antibody (Dako) for $30 \mathrm{~min}$ at $37^{\circ} \mathrm{C}$, and then with anti-mouse immunoglobulins/biotinylated (Dako) for $30 \mathrm{~min}$ at $37^{\circ} \mathrm{C}$. Following incubation with the antibodies, the bound antibody complex was visualized by incubation with streptavidin and 3,3-diaminobenzidine tetrahydrochloride. Tumor sections adjacent to those used for the immunostaining were stained with HE to exclude 
necrotic/apoptotic regions in ROIs on IHC images. IHC images were captured using a Biozero fluorescence microscope (BZ-8000; Keyence). The size of the IHC images was 2.32 pixels $/ \mu \mathrm{m}$.

The uptake of pimonidazole and the expression of HK-II were studied in the formalin-fixed, paraffin-embedded tumor sections. Two adjacent tumor sections were used for pimonidazole or HK-II staining. Five- $\mu \mathrm{m}$ adjacent sections were prepared using a Leika RM2265 microtome (Leica Microsystems). All paraffin-embedded sections were deparaffinized prior to antigen retrieval. For pimonidazole staining, the sections were stained similarly to the frozen sections, except in antigen retrieval. The antigen retrieval method for pimonidazole staining involved immersion in a citrate buffer solution ( $\mathrm{pH}$ 6.0) and heating for $15 \mathrm{~min}$ at $121^{\circ} \mathrm{C}$. For HK-II staining, the slides were immersed in a protease $\mathrm{K}$ solution (Dako) for $10 \mathrm{~min}$ at $37^{\circ} \mathrm{C}$. The slides were then immersed in $0.3 \%$ hydrogen peroxide for $10 \mathrm{~min}$. Thereafter, the slides were incubated with an anti-HK-II antibody (Chemicon International) for $30 \mathrm{~min}$ at $37^{\circ} \mathrm{C}$, and then with anti-rabbit immunoglobulins/biotinylated (Dako) for $30 \mathrm{~min}$ at $37^{\circ} \mathrm{C}$. Following antibody incubation, the bound antibody complex was visualized by incubation with streptavidin and 3,3-diaminobenzidine tetrahydrochloride (Dako).

Image analysis. [F-18]-FMISO ARG images were compared with [C-14]-FDG ARG images and the IHC images of pimonidazole, Glut-1 and $\mathrm{Ki}-67$ in the frozen sections. [F-18]-FMISO ARG images were divided into the regions of high [F-18]-FMISO uptake (FMISO+) and low [F-18]-FMISO uptake (FMISO-). The tumor-to-muscle (T/M) ratio of 4 was used as a cutoff value between FMISO+ and FMISO-, based on a previous report stating that the $\mathrm{T} / \mathrm{M}$ ratio of [F-18]-FMISO uptake was $4.4 \pm 1.33 \mathrm{~h}$ following injection of [F-18]-FMISO to Walker 256 rat carcinosarcoma (27). The [F-18]-FMISO uptake level in the calf muscles around the tumor was measured. Three ROIs $\left(0.04 \mathrm{~mm}^{2}\right)$ were assigned to the muscle and the [F-18]-FMISO uptake level was determined in these ROIs. Large necrotic/apoptotic regions were excluded from the evaluation by referring to the sections stained in HE images. Initially, we set $0.16-\mathrm{mm}^{2}$ areas in the entire FMISO+ and FMISO-. Then, we assigned random numbers to all the $0.16-\mathrm{mm}^{2}$ areas. An individual not related to our study selected seven numbers from each random number set of FMISO+ and FMISO-. The areas that were assigned the selected numbers were determined as ROIs (Fig. 1). The [F-18]-FMISO uptake level in FMISO+ was significantly higher compared to FMISO- (FMISO+, $0.45 \pm 0.11 \% \mathrm{ID} / \mathrm{g} / \mathrm{kg}$; FMISO-, $0.18 \pm 0.04 \% \mathrm{ID} / \mathrm{g} / \mathrm{kg} ; \mathrm{P}<0.001)$. The ROIs placed on the [C-14]-FDG ARG images were transferred from the [F-18]-FMISO ARG images using Fujifilm MultiGauge imaging software (Fujifilm Inc.). We manually colocalized ARG images with each other using MultiGauge. Initially, the ROIs placed on the [C-14]-FDG ARG images of $20-\mu \mathrm{m}$ sections were transferred from the [C-14]-FDG ARG images of 5- $\mu \mathrm{m}$ sections by superimposing the margins of the tumor. Subsequently, the ROIs placed on the [F-18]-FMISO ARG images of $20-\mu \mathrm{m}$ sections were transferred from the [C-14]-FDG ARG images of $20-\mu \mathrm{m}$ sections by measuring the position of the phosphor imaging plates. IHC images were compared with [C-14]-FDG ARG images by Adobe Photoshop (Adobe Systems). The positions of the ROIs on the [C-14]-FDG

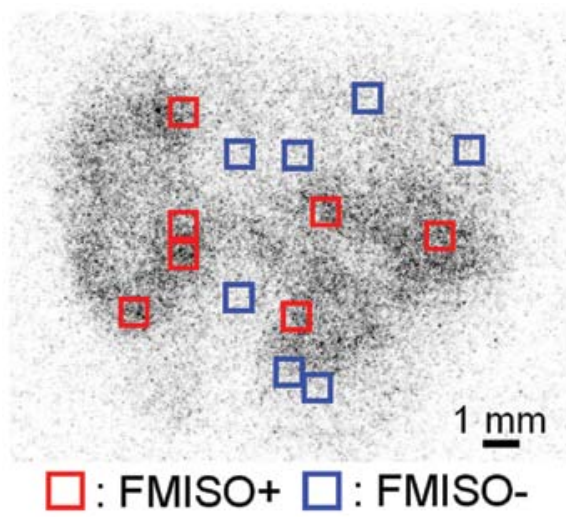

Figure 1. [F-18]-FMISO ARG image. ROIs were placed on [F-18]-FMISO ARG image to cover FMISO+ and FMISO-, except for large necrotic/apoptotic regions.

ARG images were assigned to the corresponding positions of ROIs placed on the IHC images, by superimposing the margins of the tumor in the [C-14]-FDG ARG images with those in the IHC images. The percentage of positively stained areas (\% positive) of pimonidazole and Glut-1 and the proliferation index of Ki-67 were quantified using ImageJ 1.41o software (National Institutes of Health). Thresholds for pimonidazole positivity and the proliferation index of $\mathrm{Ki}-67$ were set above the background staining using a binary image.

The IHC images of pimonidazole in paraffin sections were compared with those of HK-II. The IHC images of pimonidazole were divided into pimonidazole-positive regions (Pimo+) and pimonidazole-negative regions (Pimo-). Pimo+ and Pimo- areas were defined as the areas containing only pimonidazole-positive and -negative cells, respectively. We compared the expression of HK-II between Pimo+ and Pimo-, rather than between FMISO+ and FMISO-, since the images of HK-II IHC staining of the frozen sections were not adequately stained for evaluation. Large necrotic/apoptotic regions were excluded from the evaluation by referring to images of the sections stained with HE. Twenty-five ROIs $\left(0.04 \mathrm{~mm}^{2}\right)$ were assigned from Pimo+ and Pimo- in a double-blind manner. The positions of the ROIs on IHC images of HK-II were assigned to the corresponding positions on IHC images of pimonidazole, using Adobe Photoshop (Adobe Systems). The $\%$ positive of pimonidazole and HK-II were quantified using ImageJ 1.41o software (National Institutes of Health). The threshold for HK-II positivity was set above the background staining using a binary image.

Statistical analyses. In the analyses between FMISO+ and FMISO-, all values were averaged in seven ROIs from each tumor section. Subsequently, five values of FMISO+ and FMISO- per rat were statistically analyzed. All values are expressed as the means \pm standard deviation. Statistical analyses were performed using a non-parametric Mann-Whitney U test to evaluate the significance of differences in values between FMISO+ and FMISO-.

In the analyses between Pimo+ and Pimo-, all values were averaged in 25 ROIs from each tumor section. Five values of Pimo+ and Pimo- per rat were then statistically analyzed. All values are expressed as the means \pm standard deviation. 

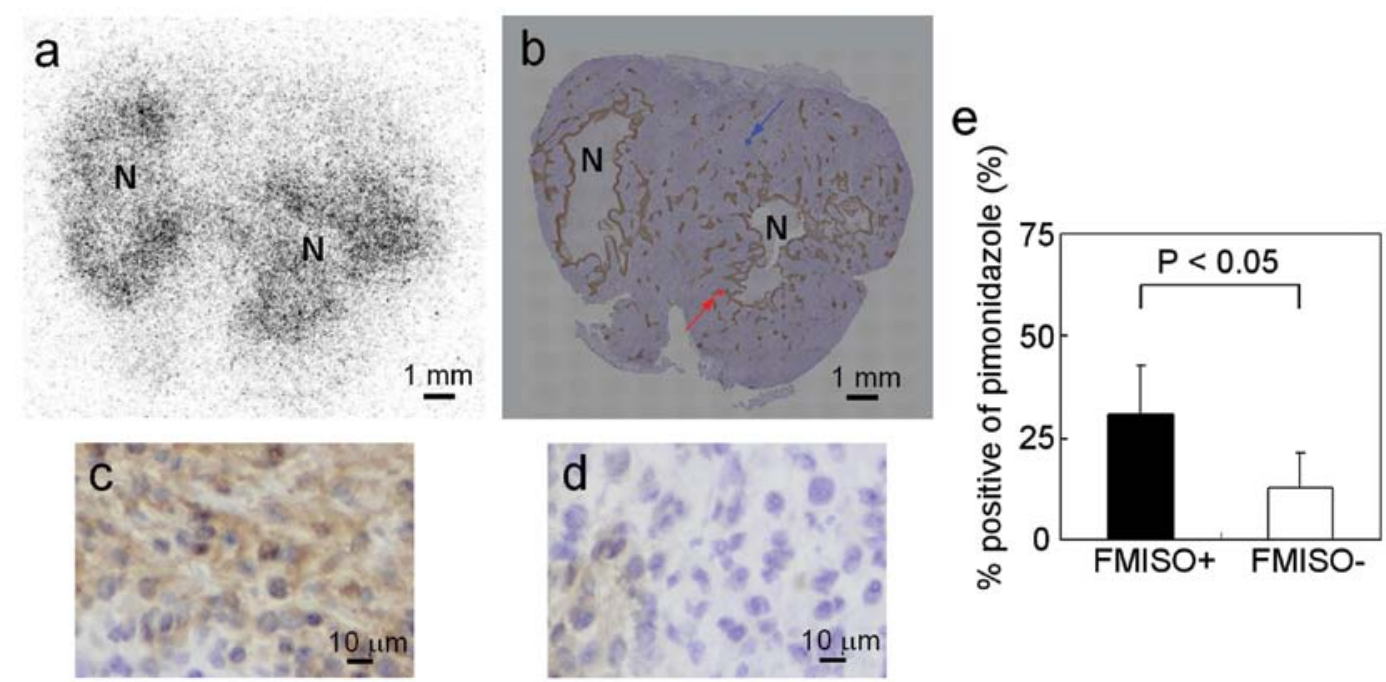

Figure 2. [F-18]-FMISO distribution in comparison with pimonidazole distribution. Representative images of (a) [F-18]-FMISO ARG and (b) pimonidazole IHC staining. Red and blue arrows denote the area of origin in panels c and d. Typical IHC stainings of pimonidazole in (c) FMISO+ and (d) FMISO(e) Pimonidazole uptake assessed by semiquantitative analysis in FMISO+ and FMISO-. N, necrotic/apoptotic regions.
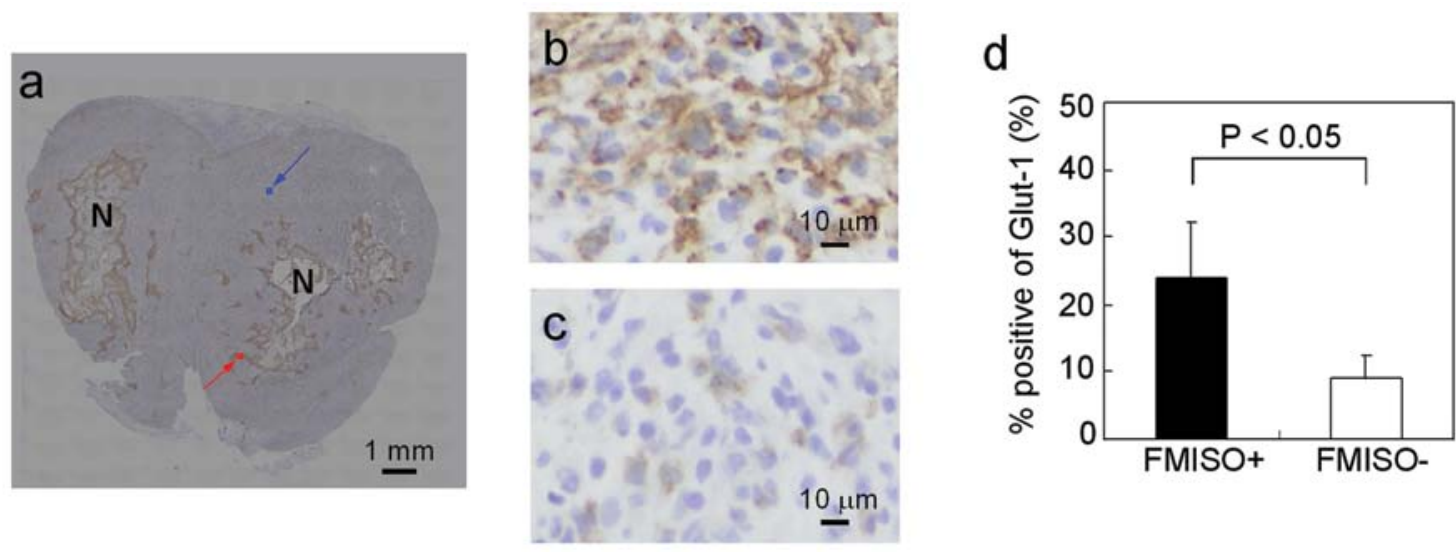

Figure 3. Glut-1 expression in comparison with [F-18]-FMISO distribution. (a) Representative image of Glut-1 IHC staining. Red and blue arrows denote the area of origin in panels b and c. Typical IHC stainings of Glut-1 in (b) FMISO+ and (c) FMISO-. (d) Glut-1 expression assessed by semiquantitative analysis in FMISO+ and FMISO-. N, necrotic/apoptotic regions.

Statistical analyses were performed using a non-parametric Mann-Whitney $U$ test to evaluate the significance of differences in values between Pimo+ and Pimo-. P-value $<0.05$ was considered to indicate a statistically significant difference. The statistical program StatView 5.0 was used for data assessment.

\section{Results}

[F-18]-FMISO distribution in comparison with pimonidazole distribution. Fig. $2 \mathrm{a}$ and $\mathrm{b}$ shows representative images of [F-18]-FMISO ARG and pimonidazole IHC staining of the whole tumor. The patterns of pimonidazole uptake were similar to those of [F-18]-FMISO uptake. The typical IHC stainings of pimonidazole in FMISO+ and FMISO- are shown in Fig. $2 \mathrm{c}$ and d, respectively. The intensity and extent of pimonidazole staining were markedly greater in FMISO+ compared to FMISO-. The results of semiquantitative analysis of pimonidazole uptake are summarized in Fig. 2e. The $\%$ positive of pimonidazole was significantly higher in
FMISO+ compared to FMISO- $(31 \pm 12 \%$ in FMISO+ and $13 \pm 9 \%$ in FMISO-; $\mathrm{P}<0.05$ ).

Glut-1 expression in comparison with [F-18]-FMISO distribution. Fig. 3a shows representative images of Glut-1 IHC staining of the whole tumor. The patterns of Glut-1 positively stained areas were similar to those of [F-18]-FMISO uptake (Fig. 2a). The typical IHC stainings of Glut-1 in FMISO+ and FMISO- are shown in Fig. $3 \mathrm{~b}$ and c, respectively. The intensity and extent of Glut-1 staining were markedly greater in FMISO+ compared to FMISO-. The results of semiquantitative analysis of Glut-1 are summarized in Fig. 3d. The \% positive of Glut-1 was significantly higher in FMISO+ compared to FMISO$(24 \pm 8 \%$ in FMISO+ and $9 \pm 4 \%$ in FMISO-, $\mathrm{P}<0.05)$.

Ki-67 expression in comparison with [F-18]-FMISO distribution. Fig. 4a shows a representative Ki-67 IHC staining of the tumor. The typical IHC stainings of Ki-67 in FMISO+ and FMISO- are shown in Fig. $4 \mathrm{~b}$ and c, respectively. Ki-67-positive 

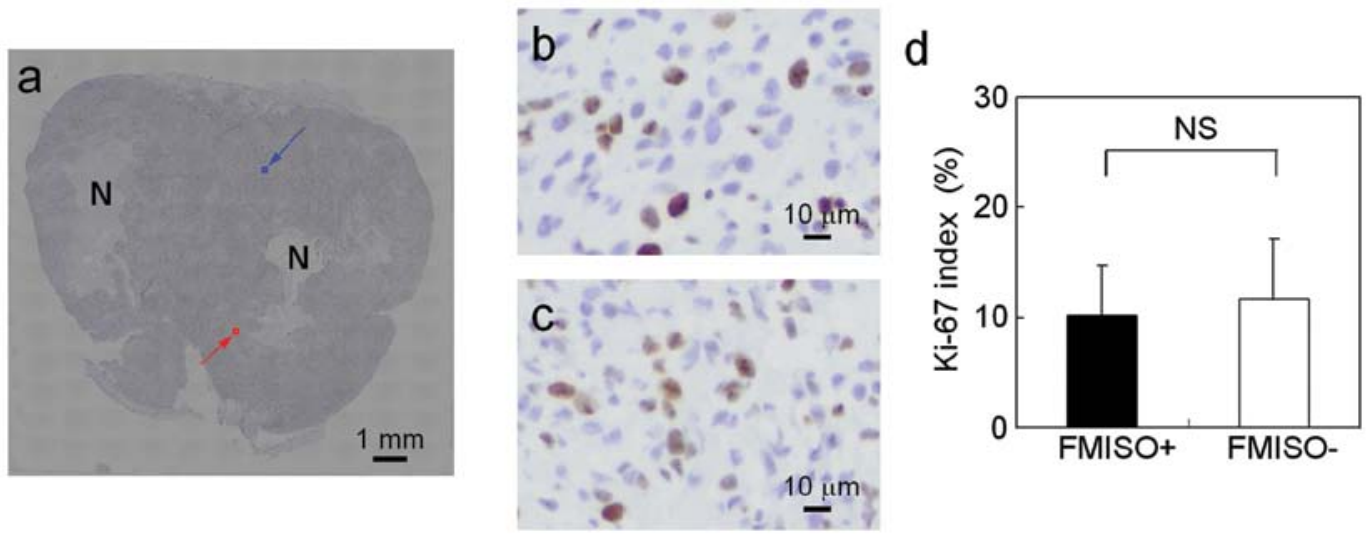

Figure 4. Ki-67 expression in comparison with [F-18]-FMISO distribution. (a) Representative image of Ki-67 IHC staining. Red and blue arrows denote the area of origin in panels b and c. Typical IHC stainings of Ki-67 in (b) FMISO+ and (c) FMISO-. (d) Ki-67 expression assessed by semiquantitative analysis in FMISO+ and FMISO-. N, necrotic/apoptotic regions.
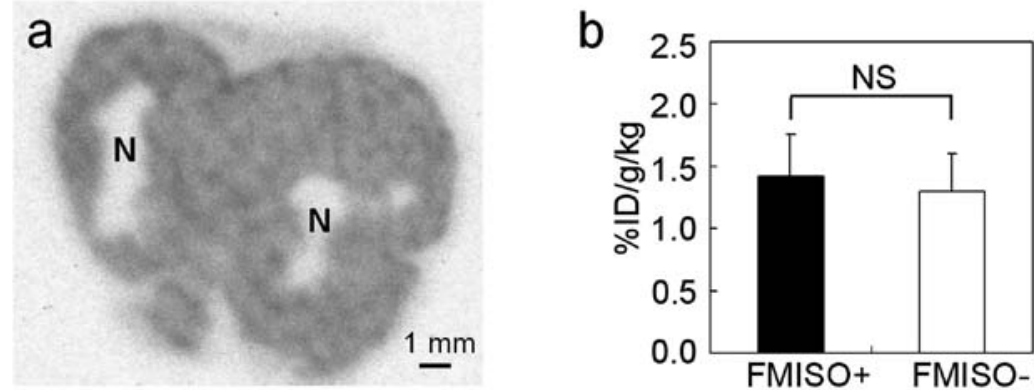

Figure 5. [C-14]-FDG distribution in comparison with [F-18]-FMISO distribution. (a) Representative image of [C-14]-FDG ARG. (b) [C-14]-FDG uptake assessed by semiquantitative analysis in FMISO+ and FMISO-. N, necrotic/apoptotic regions.
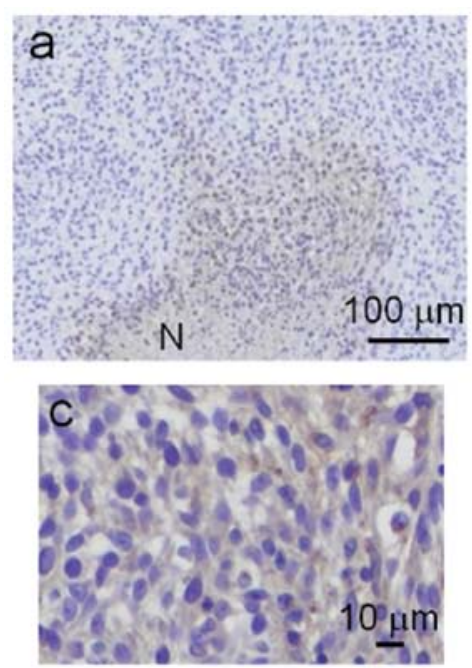
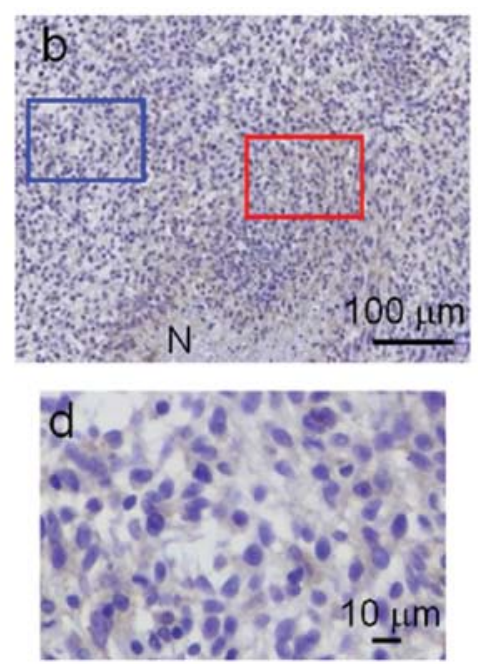

\section{e}

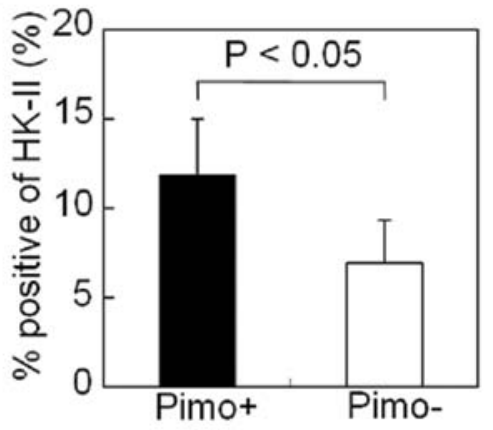

Figure 6. HK-II expression in comparison with pimonidazole distribution. Representative images of (a) pimonidazole and (b) HK-II IHC staining. Typical immunostaining of pimonidazole in (c) Pimo+ and (d) Pimo-. (e) HK-II expression assessed by semiquantitative analysis in Pimo+ and Pimo-. N, necrotic/apoptotic regions.

cells were observed in both FMISO+ and FMISO-. The results of semiquantitative analysis of $\mathrm{Ki}-67$ are summarized in Fig. 4d. There were no significant differences in Ki-67 index between FMISO+ and FMISO- $(10 \pm 5 \%$ in FMISO+ and $12 \pm 5 \%$ in FMISO-; $\mathrm{P}=\mathrm{ns})$.
[C-14]-FDG distribution in comparison with [F-18]-FMISO distribution. Fig. 5a shows a representative [C-14]-FDG ARG image of the tumor. [C-14]-FDG accumulation was observed in the entire tumor except in necrotic/apoptotic regions. The results of semiquantitative analysis of [C-14]-FDG accumula- 
tion based on ARG images are summarized in Fig. 5b. There were no significant differences in [C-14]-FDG uptake between FMISO+ and FMISO- $(1.4 \pm 0.3 \% \mathrm{ID} / \mathrm{g} / \mathrm{kg}$ in FMISO+ and $1.3 \pm 0.3 \% \mathrm{ID} / \mathrm{g} / \mathrm{kg}$ in FMISO-; $\mathrm{P}=\mathrm{ns})$.

HK-II expression in comparison with pimonidazole distribution. Fig. 6a shows representative images of pimonidazole IHC staining of the tumor. Fig. $6 \mathrm{~b}$ shows representative images of HK-II IHC staining of the tumor. The staining pattern of pimonidazole-positive areas was similar to that of HK-II-positive areas. The typical IHC stainings of HK-II in Pimo+ and Pimo- are shown in Fig. 6c and d, respectively. The intensity and extent of HK-II staining were markedly greater in Pimo+ compared to Pimo-. The results of semiquantitative analysis of HK-II are summarized in Fig. 6e. The \% positive of HK-II was significantly higher in Pimo+ compared to Pimo- $(12 \pm 3 \%$ in Pimo+ and 7 $\pm 2 \%$ in Pimo-; $\mathrm{P}<0.05)$.

\section{Discussion}

In this study, the $\%$ positive of pimonidazole and Glut-1 were higher in FMISO+ than in FMISO-. However, there were no significant differences in Ki-67 index and [C-14]-FDG uptake between FMISO+ and FMISO-. These findings indicate that intratumoral [F-18]-FMISO distribution reflects tumor hypoxia and expression of the hypoxia-related gene product Glut-1; it does not, however, reflect tumor proliferation or glucose metabolism. Radiosensitivity depends on oxygen levels in tumor tissues (2). Hypoxia imaging using [F-18]-FMISO is therefore considered useful for patient selection prior to and during radiotherapy (8-10). Moreover, biological characteristics affect radiosensitivity and radiotherapy outcome $(4,13,14)$. Therefore, [F-18]-FMISO may be used to identify hypoxic radioresistant regions among regions with similar proliferative activity and glucose metabolism activity. Such regions may be targets for dose escalation using IMRT. Our results may thus provide critical information on radiotherapy, since patients suffering from malignant glioma are generally treated by fractionated radiation therapy (28).

Our study indicated that [F-18]-FMISO distribution was similar to pimonidazole distribution and that Glut-1 expression level was higher in FMISO+. Since pimonidazole uptake is closely related to tumor hypoxia, pimonidazole has been used as an exogenous marker of radioresistance in clinical practice. Kaanders et al demonstrated that locoregional tumor control and disease-free survival were significantly reduced in patients who had head-and-neck tumors with high pimonidazole binding levels (29). These differences were not observed in the subgroup of patients undergoing accelerated radiotherapy combined with carbogen and nicotinamide (ARCON) treatment. This indicated that pimonidazole binding reflects hypoxic radiation resistance. While pimonidazole is used as the exogenous marker, Glut-1 has been used as an endogenous marker. Airley et al investigated the relationship between Glut-1 expression in cervical tumors and the prognosis following treatment of these tumors with radiotherapy (30). A high Glut-1 staining intensity in tumors indicated a shorter metastasis-free survival. This suggested that Glut-1 expression may be a potential marker of radioresistance. Therefore, the increases in pimonidazole uptake and Glut-1 expression level in FMISO+ suggest that tumor cells in FMISO+ may be more radioresistant compared to those in FMISO-.

Cellular proliferation and glucose metabolism are indicators of biological aggressiveness. Therefore, tumor areas with a high cellular proliferation or glucose metabolism may be an important target for radiotherapy, comparable with hypoxic tumor areas. Tumor hypoxia may correlate with cellular proliferation and glucose metabolism, since HIF-1 upregulates genes that induce cellular proliferation and glucose metabolism. We confirmed that the Glut-1 expression level was increased in FMISO+. Moreover, we discovered that the HK-II expression level was higher in high-pimonidazole-uptake regions (Pimo+) than in low-pimonidazole-uptake regions (Pimo-) (Fig. 6). However, we observed no significant differences in cellular proliferation and glucose metabolism between FMISO+ and FMISO-. Several studies have demonstrated discordant results regarding the correlation between hypoxia and cellular proliferation, or between hypoxia and glucose metabolism (4,5,31-36). The expressions of Glut-1 and HK-II are important factors that induce glucose metabolism $(18,19)$. However, our data demonstrated that glucose metabolism in FMISO+ was not significantly enhanced, even when the expression levels of Glut-1 in FMISO+ and HK-II in Pimo+ were increased. There was also no notable increase in cellular proliferative activity. Therefore, it is hypothesized that factors other than Glut-1 and HK-II expression may predominantly affect glucose metabolism and cellular proliferation in FMISO+. For example, the delivery of glucose is reduced in hypoxic regions due to their long distance from blood vessels. The reduced glucose delivery may result in decreased glucose metabolism and cellular proliferation.

Riesterer et al demonstrated that [F-18]-FMISO distribution in tumors is similar to the distribution of Glut-1-positive regions in the mouse mammary tumor model (37), which is consistent with our results in the glioma rat model. Regarding the correlation between oxygen concentration and proliferative activity, the proliferative activity in hypoxic regions was decreased in several types of tumors $(5,38)$. However, Evans et al reported that the hypoxia probe EF5-binding regions with a proximity to $\mathrm{Ki}-67$-positive cells (approximately $50 \mu \mathrm{m}$ ) were the $75.6 \%$ of all EF5-binding regions in human glioblastoma, suggesting that the majority of the hypoxic regions overlap with highly proliferative regions in human glioblastoma (4). Therefore, the correlation between oxygen concentration and proliferative activity is considered to differ among different types of tumors and this correlation in C6 glioma has not been fully investigated. We discovered that the proliferative activity in FMISO+ was not significantly different from that in FMISO- in C6 glioma. Regarding the correlation between oxygen concentration and glucose metabolism, hypoxia probe uptake in tumors was weakly or not correlated with glucose metabolism in several types of tumors $(31,39,40)$. In C6 glioma, however, the correlation has not been elucidated. Our results in C6 glioma also demonstrated a weak or no correlation between [F-18]FMISO distribution and glucose metabolism.

Although [F-18]-FMISO imaging may be useful for radiotherapy planning, several issues remain to be addressed. First, it is difficult to discriminate hypoxic from normoxic regions due to the low resolution of clinical PET. Furthermore, hypoxic regions in tumors were reported to be unstable (41). To optimize radiotherapy planning by [F-18]-FMISO imaging, 
[F-18]-FMISO-positive regions must match hypoxic regions at the time of radiation delivery.

There is an increasing interest in incorporating functional and molecular information into radiotherapy. Molecular imaging, including [F-18]-FDG and [F-18]-FMISO PET/CT, can provide such information (42). At present, radiotherapy planning using [F-18]-FDG and [F-18]-FMISO PET/CT is mostly conducted without detailed biological information regarding probe distribution. It is critical to elucidate the biology underlying molecular imaging. Our results provide useful insight into radiotherapy planning using [F-18]-FMISO PET, although further studies are required to determine the optimal use of such biological information in radiotherapy planning.

In conclusion, intratumoral [F-18]-FMISO distribution reflected tumor hypoxia and the expression of the hypoxia-related gene product Glut-1; it did not, however, reflect tumor proliferation or glucose metabolism. Our findings may help elucidate the biological characteristics of intratumoral [F-18]-FMISO distribution that are relevant to radiotherapy planning.

\section{Acknowledgements}

This study was partially supported by the 'Project for Developing Innovation Systems: Creation of Innovation Centers for Advanced Interdisciplinary Research Areas Program' from the Ministry of Education, Culture, Sports, Science and Technology, the Japanese Government. This study was also partially supported by a Grant-in-Aid for General Scientific Research from the Japan Society for the Promotion of Science. The authors thank the staff of the Nuclear Medicine and Central Institute of Isotope Science, Hokkaido University and the staff of the Central Research Laboratory, Hitachi, Ltd., for their support.

\section{References}

1. Brown JM and Giaccia AJ: The unique physiology of solid tumors: opportunities (and problems) for cancer therapy. Cancer Res 58: 1408-1416, 1998.

2. Gray LH, Conger AD, Ebert M, Hornsey S and Scott OC: The concentration of oxygen dissolved in tissues at the time of irradiation as a factor in radiotherapy. Br J Radiol 26: 638-648, 1953.

3. Höckel M and Vaupel P: Tumor hypoxia: definitions and current clinical, biologic, and molecular aspects. J Natl Cancer Inst 93: 266-276, 2001.

4. Evans SM, Jenkins KW, Chen HI, Jenkins WT, Judy KD, Hwang WT, Lustig RA, Judkins AR, Grady MS, Hahn SM and Koch CJ: The relationship among hypoxia, proliferation, and outcome in patients with de novo glioblastoma: A pilot study. Transl Oncol 3: 160-169, 2010.

5. Evans SM, Hahn SM, Magarelli DP and Koch CJ: Hypoxic heterogeneity in human tumors: EF5 binding, vasculature, necrosis, and proliferation. Am J Clin Oncol 24: 467-472, 2001.

6. Troost EG, Laverman P, Philippens ME, et al: Correlation of $\left[{ }^{18} \mathrm{~F}\right] \mathrm{FMISO}$ autoradiography and pimonidazole immunohistochemistry in human head and neck carcinoma xenografts. Eur J Nucl Med Mol Imaging 35: 1803-1811, 2008.

7. Troost EG, Laverman P, Kaanders JH, Philippens M, Lok J, Oyen WJ, van der Kogel AJ, Boerman OC and Bussink J: Imaging hypoxia after oxygenation-modification: comparing $\left[{ }^{18} \mathrm{~F}\right] \mathrm{FMISO}$ autoradiography with pimonidazole immunohistochemistry in human xenograft tumors. Radiother Oncol 80: 157-164, 2006.

8. Spence AM, Muzi M, Swanson KR, O'Sullivan F, Rockhill JK, Rajendran JG, Adamsen TC, Link JM, Swanson PE, Yagle KJ, Rostomily RC, Silbergeld DL and Krohn KA: Regional hypoxia in glioblastoma multiforme quantified with $\left[{ }^{18} \mathrm{~F}\right]$ fluoromisonidazole positron emission tomography before radiotherapy: correlation with time to progression and survival. Clin Cancer Res 14: 2623-2630, 2008.
9. Eschmann SM, Paulsen F, Reimold M, Dittmann H, Welz S, Reischl G, Machulla HJ and Bares R: Prognostic impact of hypoxia imaging with ${ }^{18} \mathrm{~F}$-misonidazole PET in non-small cell lung cancer and head and neck cancer before radiotherapy. J Nucl Med 46: 253-260, 2005.

10. Rajendran JG, Schwartz DL, O'Sullivan J, Peterson LM, Ng P, Scharnhorst J, Grierson JR and Krohn KA: Tumor hypoxia imaging with [F-18] fluoromisonidazole positron emission tomography in head and neck cancer. Clin Cancer Res 12: 5435-5441, 2006.

11. Overgaard J: Clinical evaluation of nitroimidazoles as modifiers of hypoxia in solid tumors. Oncol Res 6: 509-518, 1994.

12. Overgaard J and Horsman MR: Modification of hypoxia-induced radioresistance in tumors by the use of oxygen and sensitizers. Semin Radiat Oncol 6: 10-21, 1996.

13. Moeller BJ and Dewhirst MW: HIF-1 and tumour radiosensitivity. Br J Cancer 95: 1-5, 2006.

14. Moeller BJ, Dreher MR, Rabbani ZN, Schroeder T, Cao Y, Li CY and Dewhirst MW: Pleiotropic effects of HIF-1 blockade on tumor radiosensitivity. Cancer Cell 8: 99-110, 2005.

15. Dang CV and Semenza GL: Oncogenic alterations of metabolism. Trends Biochem Sci 24: 68-72, 1999.

16. Kamlah F, Eul BG, Li S, Lang N, Marsh LM, Seeger W, Grimminger F, Rose F and Hänze J: Intravenous injection of siRNA directed against hypoxia-inducible factors prolongs survival in a Lewis lung carcinoma cancer model. Cancer Gene Ther 16: 195-205, 2009.

17. Le QT and Courter D: Clinical biomarkers for hypoxia targeting. Cancer Metastasis Rev 27: 351-362, 2008

18. Reske SN, Grillenberger KG, Glatting G, Port M, Hildebrandt M, Gansauge F and Beger HG: Overexpression of glucose transporter 1 and increased FDG uptake in pancreatic carcinoma. J Nucl Med 38: 1344-1348, 1997.

19. Aloj L, Caracó C, Jagoda E, Eckelman WC and Neumann RD: Glut-1 and hexokinase expression: relationship with 2-fluoro2-deoxy-D-glucose uptake in A431 and T47D cells in culture. Cancer Res 59: 4709-4714, 1999.

20. Semenza GL: Targeting HIF-1 for cancer therapy. Nat Rev Cancer 3: 721-732, 2003.

21. Zhao S, Kuge Y, Yi M, Zhao Y, Hatano T, Magota K, Nishijima K, Kohanawa M and Tamaki N: Dynamic 11C-methionine PET analysis has an additional value for differentiating tumors from granulomas: An experimental study using small animal PET. Eur J Nucl Med Mol Imaging 38: 1876-1886, 2011.

22. Tang G, Wang M, Tang X, Gan M and Luo L: Fully automated one-pot synthesis of $\left[{ }^{18} \mathrm{~F}\right]$ fluoromisonidazole. Nucl Med Biol 32: 553-558, 2005.

23. Oh SJ, Chi DY, Mosdzianowski C, Kim JY, Gil HS, Kang SH, Ryu JS and Moon DH: Fully automated synthesis of $\left[{ }^{18} \mathrm{~F}\right]$ fluoromisonidazole using a conventional $\left[{ }^{18} \mathrm{~F}\right] \mathrm{FDG}$ module. Nucl Med Biol 32: 899-905, 2005.

24. Brown RS, Leung JY, Fisher SJ, Frey KA, Ethier SP and Wahl RL: Intratumoral distribution of tritiated fluorodeoxyglucose in breast carcinoma: I. Are inflammatory cells important? J Nucl Med 36: 1854-1861, 1995.

25. Toyama H, Ichise M, Liow JS, Modell KJ, Vines DC, Esaki T, Cook M, Seidel J, Sokoloff L, Green MV and Innis RB: Absolute quantification of regional cerebral glucose utilization in mice by ${ }^{18}$ F-FDG small animal PET scanning and $2-{ }^{14} \mathrm{C}-\mathrm{DG}$ autoradiography. J Nucl Med 45: 1398-1405, 2004.

26. Hatanaka Y, Imaoka Y, Torisu K, Kamihara Y, Hashizume K, Ichimura K, Yoshino T and Tani Y: A simplified, sensitive immunohistochemical detection system employing signal amplification based on fluorescyl-tyramide/antifluorescein antibody reaction: its application to pathologic testing and research. Appl Immunohistochem Mol Morphol 16: 87-93, 2008.

27. Sorger D, Patt M, Kumar P, Wiebe LI, Barthel H, Seese A, Dannenberg C, Tannapfel A, Kluge R and Sabri O: $\left[{ }^{18} \mathrm{~F}\right]$ Fluoroazomycinarabinofuranoside $\left({ }^{18} \mathrm{FAZA}\right)$ and $\left[{ }^{18} \mathrm{~F}\right]$ Fluoromisonidazole ( $\left.{ }^{18} \mathrm{FMISO}\right)$ : a comparative study of their selective uptake in hypoxic cells and PET imaging in experimental rat tumors. Nucl Med Biol 30: 317-326, 2003.

28. Buatti J, Ryken TC, Smith MC, Sneed P, Suh JH, Mehta M and Olson JJ: Radiation therapy of pathologically confirmed newly diagnosed glioblastoma in adults. J Neurooncol 89: 313-337, 2008.

29. Kaanders JH, Wijffels KI, Marres HA, Ljungkvist AS, Pop LA, van den Hoogen FJ, de Wilde PC, Bussink J, Raleigh JA and van der Kogel AJ: Pimonidazole binding and tumor vascularity predict for treatment outcome in head and neck cancer. Cancer Res 62: 7066-7074, 2002. 
30. Airley R, Loncaster J, Davidson S, Bromley M, Roberts S, Patterson A, Hunter R, Stratford I and West C: Glucose transporter glut-1 expression correlates with tumor hypoxia and predicts metastasis-free survival in advanced carcinoma of the cervix. Clin Cancer Res 7: 928-934, 2001.

31. Thorwarth D, Eschmann SM, Holzner F, Paulsen F and Alber M Combined uptake of $\left[{ }^{18} \mathrm{~F}\right]$ FDG and $\left[{ }^{18} \mathrm{~F}\right]$ FMISO correlates with radiation therapy outcome in head-and-neck cancer patients. Radiother Oncol 80: 151-156, 2006.

32. Wyss MT, Honer M, Schubiger PA and Ametamey SM: NanoPET imaging of $\left[{ }^{18} \mathrm{~F}\right]$ fluoromisonidazole uptake in experimental mouse tumours. Eur J Nucl Med Mol Imaging 33: 311-318, 2006.

33. Zimny M, Gagel B, DiMartino E, Hamacher K, Coenen HH, Westhofen M, Eble M, Buell U and Reinartz P: FDG - a marker of tumour hypoxia? A comparison with $\left[{ }^{18} \mathrm{~F}\right]$ fluoromisonidazole and $\mathrm{pO}_{2}$-polarography in metastatic head and neck cancer. Eur $\mathbf{J}$ Nucl Med Mol Imaging 33: 1426-1431, 2006.

34. Gagel B, Piroth M, Pinkawa M, Reinartz P, Zimny M, Kaiser HJ, Stanzel S, Asadpour B, Demirel C, Hamacher K, Coenen HH, Scholbach T, Maneschi P, DiMartino E and Eble MJ: pO 2 polarography, contrast enhanced color duplex sonography (CDS), $\left[{ }^{18} \mathrm{~F}\right]$ fluoromisonidazole and $\left[{ }^{18} \mathrm{~F}\right]$ fluorodeoxyglucose positron emission tomography: validated methods for the evaluation of therapy-relevant tumor oxygenation or only bricks in the puzzle of tumor hypoxia? BMC Cancer 7: 113, 2007.

35. Rajendran JG, Mankoff DA, O'Sullivan F, Peterson LM Schwartz DL, Conrad EU, Spence AM, Muzi M, Farwell DG and Krohn KA: Hypoxia and glucose metabolism in malignant tumors: evaluation by $\left.{ }^{18} \mathrm{~F}\right]$ fluoromisonidazole and $\left[{ }^{18} \mathrm{~F}\right]$ fluorodeoxyglucose positron emission tomography imaging. Clin Cancer Res 10: 2245-2252, 2004.
36. Cherk MH, Foo SS, Poon AM, Knight SR, Murone C, Papenfuss AT, Sachinidis JI, Saunder TH, O'Keefe GJ and Scott AM: Lack of correlation of hypoxic cell fraction and angiogenesis with glucose metabolic rate in non-small cell lung cancer assessed by ${ }^{18} \mathrm{~F}$-Fluoromisonidazole and ${ }^{18} \mathrm{~F}$-FDG PET. J Nucl Med 47: 1921-1926, 2006.

37. Riesterer O, Honer M, Jochum W, Oehler C, Ametamey S and Pruschy M: Ionizing radiation antagonizes tumor hypoxia induced by antiangiogenic treatment. Clin Cancer Res 12: 3518-3524, 2006.

38. Wijffels KI, Marres HA, Peters JP, Rijken PF, van der Kogel AJ and Kaanders JH: Tumour cell proliferation under hypoxic conditions in human head and neck squamous cell carcinomas. Oral Oncol 44: 335-344, 2008.

39. Rajendran JG, Wilson DC, Conrad EU, Peterson LM, Bruckner JD, Rasey JS, Chin LK, Hofstrand PD, Grierson JR, Eary JF and Krohn KA: $\left[{ }^{18} \mathrm{~F}\right] \mathrm{FMISO}$ and $\left[{ }^{18} \mathrm{~F}\right] \mathrm{FDG}$ PET imaging in soft tissue sarcomas: correlation of hypoxia, metabolism and VEGF expression. Eur J Nucl Med Mol Imaging 30: 695-704, 2003.

40. Kubota K, Tada M, Yamada S, Hori K, Saito S, Iwata R, Sato K, Fukuda $\mathrm{H}$ and Ido T: Comparison of the distribution of fluorine-18 fluoromisonidazole, deoxyglucose and methionine in tumour tissue. Eur J Nucl Med 26: 750-757, 1999.

41. Dewhirst MW: Intermittent hypoxia furthers the rationale for hypoxia-inducible factor-1 targeting. Cancer Res 67: 854-855, 2007.

42. Søvik A, Malinen E and Olsen DR: Strategies for biologic imageguided dose escalation: a review. Int J Radiat Oncol Biol Phys 73: $650-658,2009$ 